\title{
Double-injection perivascular ultrasound-guided axillary brachial plexus block according to needle positioning: 12 versus 6 o'clock position of the axillary artery
}

\author{
Sooyoung Cho, Youn Jin Kim, Jong-Hak Kim, and Hee-Jung Baik \\ Department of Anesthesiology and Pain Medicine, Ewha Womans University School of Medicine, Seoul, Korea
}

Background: We conducted prospective, randomized, observer-blinded trial to compare two double-injection perivascular (PV) ultrasound-guided techniques of axillary brachial plexus block (BPB).

Methods: American Society of Anesthesiologists physical status I-II, 50 patients undergoing surgery of the forearm, wrist or hand were randomly allocated to two groups. For PV12 group, injection was carried out at the 12 o'clock position using $24 \mathrm{ml}$ of 2\% lidocaine. Patients of PV6 group got their injection of $24 \mathrm{ml}$ of $2 \%$ lidocaine at direction of 6 o'clock of axillary artery. For all 2 groups, the musculocutaneous nerve was identified and $5 \mathrm{ml}$ of $2 \%$ lidocaine was deposited around the nerve. The performance time and the onset time were recorded. The induction time (sum of performance and onset time), the success rate of the block, the need rate of rescue block, and incidence of adverse events was compared.

Results: The success rate was same (84\%) in two groups. The performance time, onset time, and induction time showed no differences between two groups. There were no differences in vessel puncture, paresthesia, and numbness.

Conclusions: Double-injection perivascular ultrasound-guided axillary BPB can be performed at 12 o'clock or 6 o'clock position of axillary artery, and performer may choose needle targeting position by considering surgery site. Thus perivascular double-injection technique may be an alternative method for axillary BPB and useful in case of difficult block. (Korean J Anesthesiol 2014; 66: 112-119)

Key Words: Axillary brachial plexus block, Perivascular, Ultrasound.

Received: April 12, 2013. Revised: 1st, May 26, 2013; 2nd, July 10, 2013. Accepted: July 29, 2013.

Corresponding author: Youn Jin Kim, M.D., Department of Anesthesiology and Pain Medicine, Ewha Womans University School of Medicine, 1071, Anyangcheon-ro, Yangcheon-gu, Seoul 158-710, Korea. Tel: 82-2-2650-5285, Fax: 82-2-2655-2924, E-mail: ankyj@ewha.ac.kr

(c) This is an open-access article distributed under the terms of the Creative Commons Attribution Non-Commercial License (http:// creativecommons.org/licenses/by-nc/3.0/), which permits unrestricted non-commercial use, distribution, and reproduction in any medium, provided the original work is properly cited. 


\section{Introduction}

Axillary brachial plexus block (BPB) performed in patients receiving hand or wrist surgery is one of the methods for regional anesthesia which allows for early postoperative recovery and discharge in outpatient surgery [1]. Recently, ultrasound-guided axillary brachial plexus block (US-ABPB) has been widely used. The injection and diffusion of a drug can be checked in real time through ultrasound, so it is helpful in reducing the dosage of local anesthetics (LA) while heightening the quality of the nerve block and the rapid onset of the blocking effect [2,3]. Using this advantage of checking the status before and after the injection of LA, studies have been done on the effect of this technique in which the LA accumulates in the axillary artery and diffuses into the surrounding nerves, rather than identifying the nerve and injecting the LA close to the nerve $[4,5]$.

In the past, Thompson and Rorie [6] recommended multiple injections of small volumes of LA rather than single injection of large volumes of LA because compartment septae exist within the axillary sheath which can become the cause for anesthetizing only certain nerves. However, the research of Partridge et al. [7] showed that the sheath has permeability so it is clinically unnecessary to inject the LA in several places. Regarding such contrasting results, Ay et al. [8] explained that although the axillary sheath is divided into compartments and a septum exists between the compartments, the injecting pressure in large volumes of LA can cause a defect in the septum causing the LA to spread through all the nerves. In fact, Imasogie et al. [4] and Bernucci et al. [5] compared perineural (PN) injection and perivascular (PV) injection reporting that there was no difference in the success rate.

Based on the fact that PV injection is as effective as PN injection, the authors of this study conducted a comparative investigation the two areas surrounding the blood vessel. The first area was anterior to the axillary artery in the 12 o'clock direction on the ultrasound image, and the other area was posterior to the axillary artery in the 6 o'clock direction on the ultrasound image. Precedent studies $[4,5]$ used a method in which LA was PV injected in the 6 o'clock direction, but the authors of this study hypothesized that injecting the LA in the 12 o'clock direction would have a better approach and cause less injury to the surrounding tissue because it is closer to the skin. Hence, the induction time, the success rate of the block, and the incidence of adverse events were compared for injections in the 6 and 12 o'clock directions.

\section{Materials and Methods}

This research was a prospective, randomized, observerblinded trial. It was approved by the Institutional Review Board of the hospital, and after explaining the purpose and methods of the research to the patients, 50 patients who provided written consent were chosen as subjects. The selected subjects were adult patients with American Society of Anesthesiologists physical status I-II, 20 years of age or older and less than 85 who were scheduled for hand or wrist surgery which could be done through axillary BPB. Pregnant patients, patients with infections in the injection area, patients with allergies to LA, patients with coagulopathy, and patients unable to communicate were excluded as subjects.

The patients were divided randomly into two groups. A total of 50 patients, 25 patients in each group, were included in the research. There were no significant differences in the demographic characteristics between the two groups (Table 1).

A computer-generated sequence of random numbers and the sealed envelope technique were used to assign the patients to the groups. The nerve block was performed by only 2 expert anesthesiologists who have performed US-ABPB 50 times or more. After the patient arrived in the operating room, the standard monitoring equipment including electrocardiogram, blood pressure, and pulse oximetry was attached. The patients were put in a supine position with their operated arms abducted at $90^{\circ}$ and externally rotated and with their elbows in the arms bent at $90^{\circ}$. The patient was sedated with IV injection of midazolam $0.05 \mathrm{mg} / \mathrm{kg}$ and fentanyl $0.5-1.0 \mu \mathrm{g} / \mathrm{kg}$, and the axillary area of the patient was disinfected with povidone-iodine solution while supplying oxygen at $6 \mathrm{~L} / \mathrm{min}$ through an oxygen mask.

The location of the nerve and artery was identified using the ultrasound equipment Sonosite M-Turbo (Sonosite ${ }^{\circledR}$, Bothell, WA, USA). The $38 \mathrm{~mm}, 7-12 \mathrm{MHz}$ linear probe of the ultrasound was covered with sterile vinyl, and located at the axillary fold of the patient so that the artery and nerve were seen in the short axis view. It was positioned so that the 3 o'clock direction faced medial and the 9 o'clock direction faced lateral according to the axillary artery on the ultrasound image. In this view, the

Table 1. Patient Characteristics

\begin{tabular}{lccc}
\hline & $\begin{array}{c}\text { PV12 } \\
(\mathrm{n}=25)\end{array}$ & $\begin{array}{c}\text { PV6 } \\
(\mathrm{n}=25)\end{array}$ & $\mathrm{P}$ \\
\hline Age $(\mathrm{yr})$ & $48.1 \pm 15.7$ & $48.2 \pm 16.3$ & 0.805 \\
Sex $(\mathrm{male} /$ female $)$ & $11 / 14$ & $15 / 10$ & 0.396 \\
Height $(\mathrm{cm})$ & $163.6 \pm 9.5$ & $166.8 \pm 11.8$ & 0.329 \\
Weight $(\mathrm{kg})$ & $60.6 \pm 10.1$ & $64.5 \pm 12.5$ & 0.257 \\
BMI $\left(\mathrm{kg} / \mathrm{m}^{2}\right)$ & $22.6 \pm 2.6$ & $23.1 \pm 3.2$ & 0.488 \\
ASA classification $(\mathrm{I} / \mathrm{II})$ & $11 / 14$ & $15 / 10$ & 0.396 \\
\hline
\end{tabular}

Values are expressed as mean \pm SD or counts. PV12: double-injection perivascularultrasound-guided axillary brachial plexus block at 12 o'clock position of the axillary artery. PV6: double-injection perivascular ultrasound-guided axillary brachial plexus block at 6 o'clock position of the axillary artery. There were no significantdifferences between groups. BMI: body mass index, ASA: American Society of Anesthesiologists. 
12 o'clock direction closer to the skin faced anteriorly, while the deeper point of the 6 o'clock direction faced posteriorly (Fig. 1). Here, the direction of each nerve was recorded. The block was performed with the in-plane technique using a 22 gauge, $50 \mathrm{~mm}$ needle (Stimuplex ${ }^{\circledR}$, B. Braun, Melsungen, Germany) connected to a syringe containing $2 \%$ lidocaine.

For the PV12 group, the needle tip was positioned anteriorly in the 12 o'clock direction of the axillary artery, and after checking that the needle was not mounted in the artery, $24 \mathrm{ml}$ of LA were injected. Here, the injection process of the LA was observed with the ultrasound, so when the position of the needle moved due to the injection of the LA, it was readjusted to be positioned anteriorly at 12 o'clock of the axillary artery (Fig. 2A).

For the PV6 group, the needle tip was positioned posteriorly in the 6 o'clock direction of the axillary artery, and after checking that the needle was not mounted in the artery, $24 \mathrm{ml}$ of LA were injected. Likewise in the PV12 group, the LA was injected while checking with ultrasound (Fig. 2B).

The musculocutaneous nerve was separately blocked in both groups. With the ultrasound, the point of the needle was positioned close to the nerve in the coracobrachialis muscle. Using a nerve stimulator (Stimuplex ${ }^{\circledR}$ Dig RC, B. Braun, Germany) an electronic stimulus of $2 \mathrm{~Hz}, 0.6 \mathrm{~mA}$ was given. When there was flexion in the elbow, the electronic stimulus was reduced to $0.5 \mathrm{~mA}$ to check whether the motor response from the stimulus was maintained. Additionally, $1 \mathrm{ml}$ of $2 \%$ lidocaine was injected slowly, and when the loss of response was confirmed, the needle was considered to be appropriately positioned, and the remaining $4 \mathrm{ml}$ of LA were administered (Fig. 2C).

After performing the block, the operating anesthesiologist re- corded the block performance time (the time taken from insertion to removal of the needle), number of needle insertions, and number of axillary arteries and veins. After completing procedure, a blind observer measured the degree of sensory and motor block and the onset time, and calculated the induction time (the time taken for the appropriate level of surgical anesthesia from the time of the needle insertion).

The degree of sensory block and motor block was evaluated according to the movement and dermatome of each nerve, and it was evaluated at 2.5 minute intervals for 15 minutes with the final needle removal point as 0 minutes. The sensory block was evaluated with the pinprick test and recorded in two stages: 0 = normal sensation; 1 = no pain sensation. The sensory block of the median nerve was evaluated on the palm side of the 3rd finger, the ulnar nerve on the palm nerve of the 5th finger, the radial nerve in the lateral portion of the back of the hand, and the musculotaneous nerve in the lateral portion of the forearm. Motor block was evaluated in the following stages: $0=$ no weakness; 1 = paresis; 2 = paralysis. Motor block of the median nerve was evaluated by flexion of the 2nd and 3rd fingers, ulnar nerve by flexion of the 4th and 5th fingers, radial nerve by abduction of the thumb, and musculocutaneous nerve by the flexion movement of the elbow.

The level of surgical anesthesia was defined as when the sensory block reached stage 1 in all 4 nerves, and motor block reached stage 1 or 2 . When the sensory block did not reach stage 1 in 15 minutes after the block procedure, it was considered to be a block failure and LA were additionally injected or changed to general anesthesia. A successful block was when there was no need for any additional block procedures or general anesthesia.
A

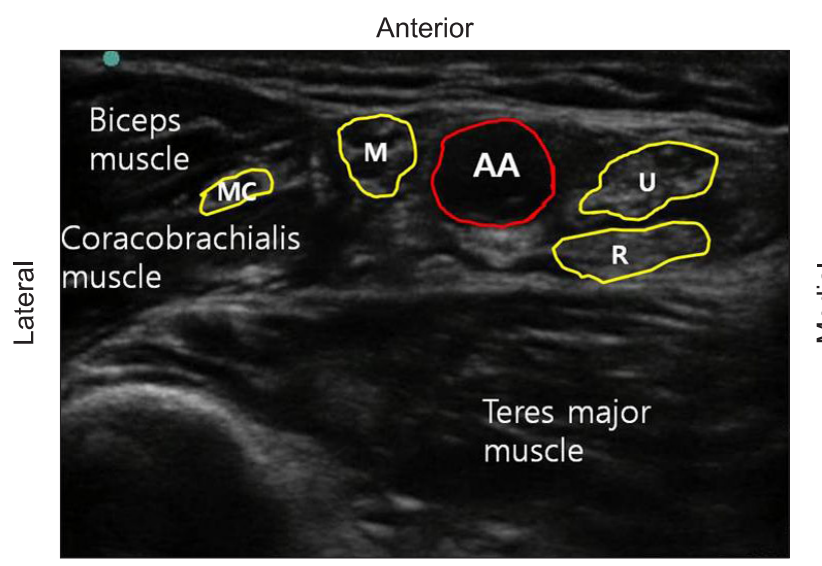

B

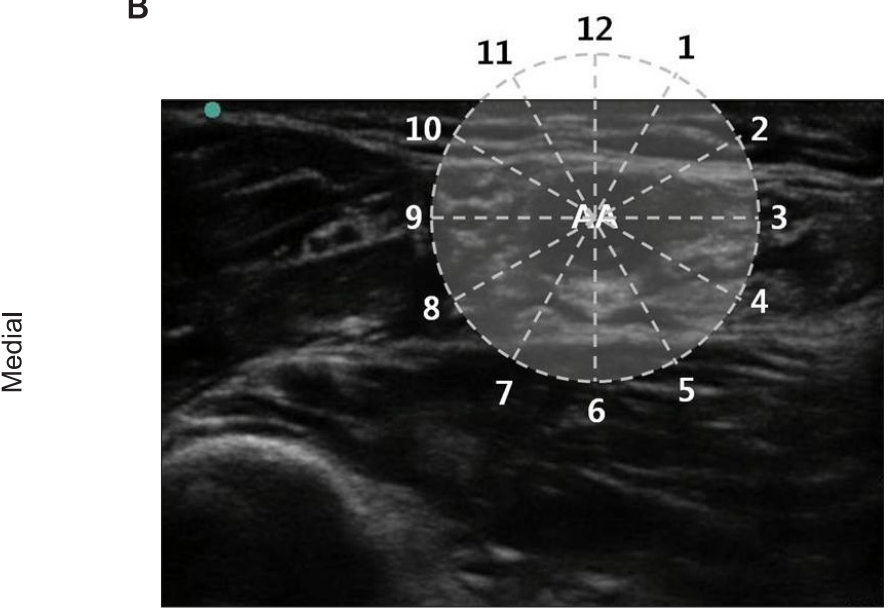

Fig. 1. (A) Schematic illustration of ultrasound scan image when the ultrasound probe was placed at the axillary crease. (B) The nerve positions were described using the clock face radiating out from the central axis of the AA. AA: axillary artery, M: median nerve, U: ulnar nerve, R: radial nerve, MC: musculocutaneous nerve. 

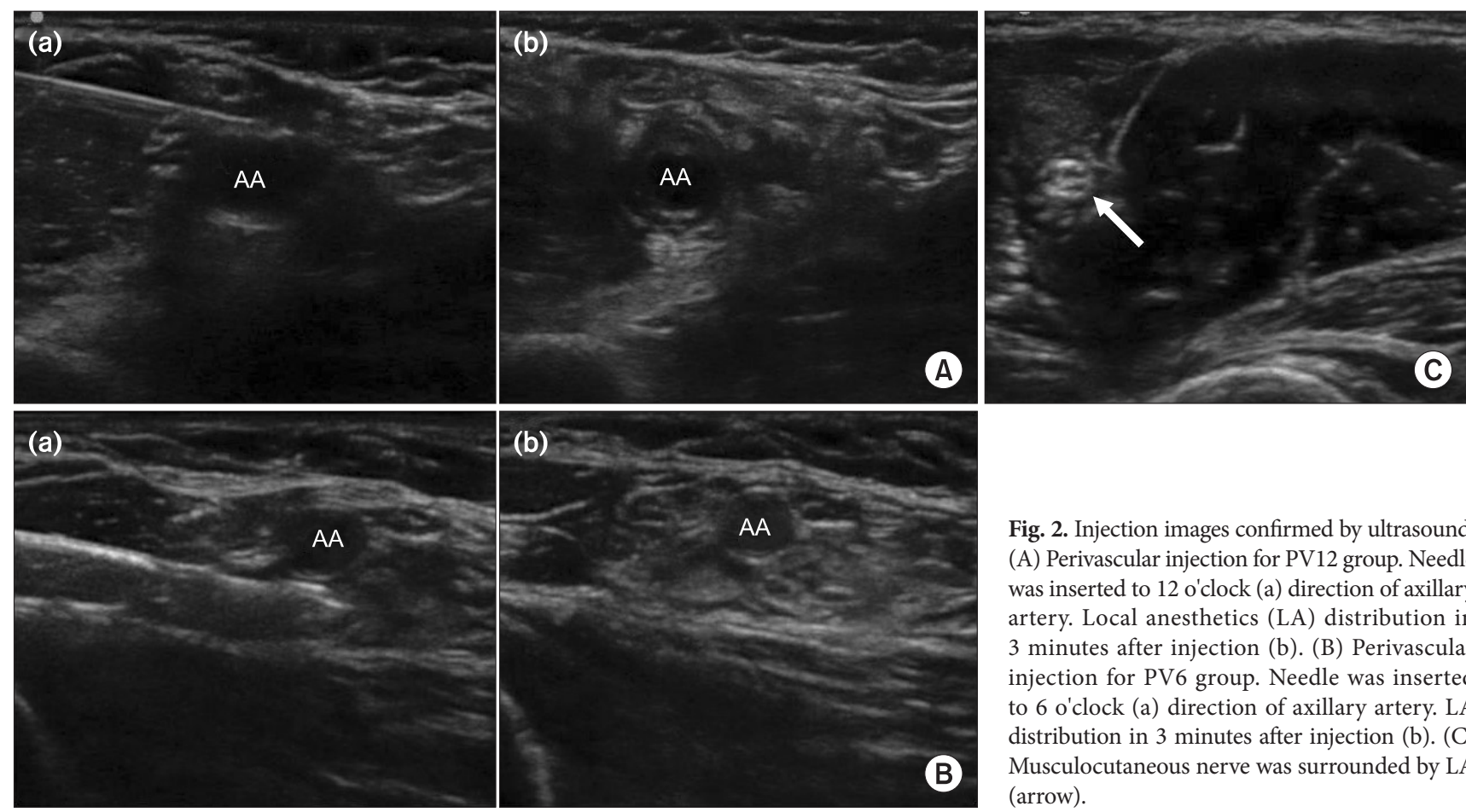

Fig. 2. Injection images confirmed by ultrasound. (A) Perivascular injection for PV12 group. Needle was inserted to 12 o'clock (a) direction of axillary artery. Local anesthetics (LA) distribution in 3 minutes after injection (b). (B) Perivascular injection for PV6 group. Needle was inserted to 6 o'clock (a) direction of axillary artery. LA distribution in 3 minutes after injection (b). (C) Musculocutaneous nerve was surrounded by LA (arrow).

Table 2. Block Performance Data

\begin{tabular}{lccc}
\hline & PV12 & PV6 \\
& $(\mathrm{n}=25)$ & $194.7 \pm 69.3$ & P \\
\hline Block performance time (sec) & $186.1 \pm 64.1$ & $385.1 \pm 166.9$ & 0.676 \\
Onset time (sec) & $357.5 \pm 200.9$ & $579.9 \pm 151.1$ & 0.630 \\
Induction time (sec) & $543.5 \pm 204.3$ & $84 \%$ & 0.516 \\
Success rate (\%) & $84 \%$ & $6.67 \pm 2.89$ & $0.33 \pm 3.65$ \\
Time to median nerve sensory block (min) & $5.95 \pm 2.56$ & $5.71 \pm 1.79$ & 0.171 \\
Time to ulnar nerve sensory block (min) & $6.90 \pm 2.95$ & 1.1 & 0.125 \\
Time to radial nerve sensory block (min) & $7.14 \pm 3.73$ & 0.65 \\
No. of needle insertion & 1.3 & & \\
\hline
\end{tabular}

Values are expressed as mean \pm SD or counts (range). PV12: double-injection perivascular ultrasound-guided axillary brachial plexus block at 12 o'clock position of the axillary artery. PV6: double-injection perivascular ultrasound-guided axillary brachial plexus block at 6 o'clock position of the axillary artery. There were no significance differences between groups.

Patient sedation during surgery was achieved by continuous infusion of propofol at $15-50 \mu \mathrm{g} / \mathrm{kg} / \mathrm{min}$, and sedation was performed to around 3-4 on the Modified Observer's Assessment of Alertness/Sedation Scale (OAA/S).

Any adverse events in the patients were evaluated while performing the block, during surgery, and after surgery. In addition, patients who had adverse events were monitored for up to 6 weeks after surgery through an outpatient clinic even after discharge. The adverse events from the toxicity of the LA were numbness of the tongue and lips, a metallic taste, tinnitus, disorientation, loss of consciousness, seizure, arrhythmia, respiratory arrest, and cardiac arrest. The occurrence of adverse events from blood vessel damage, such as hematoma, and from nerve damage, such as paresthesia and numbness, were monitored.

Data management and all statistical analyses were done with SPSS version 20.0 (SPSS Inc, Chicago, Illinois, USA). Student t-test was used for continuous variables, while the chi-square test was used for categorical variables to analyze the differences between the two groups. All tests results were considered to be significant when the P value was less than 0.05 .

\section{Results}

Twenty-five patients were assigned to each group, and when observed for 15 minutes after the block, 21 patients each in the PV12 and PV6 group reached surgical anesthesia, respectively, 
so the success rate for both groups was $84 \%$. The mean block performance time was 186.1 and 194.7 seconds for the PV12 and PV6 group, respectively, so there was no statistical difference $(P=0.676)$. The mean onset time was 357.5 and 385.1 seconds, respectively, so there was no significant difference between

Table 3. Anatomy and Complications

\begin{tabular}{lccc}
\hline & $\begin{array}{c}\text { PV12 } \\
(\mathrm{n}=25)\end{array}$ & $\begin{array}{c}\text { PV6 } \\
(\mathrm{n}=25)\end{array}$ & $\mathrm{P}$ \\
\hline Axillary artery & $1.08 \pm 0.28$ & $1.00 \pm 0.00$ & 0.155 \\
Axillary vein & $2.48 \pm 1.36$ & $2.16 \pm 0.94$ & 0.338 \\
Complication & & & \\
LA toxicity & 0 & 0 & 0.552 \\
Vascular puncture & 1 & 2 & \\
Paresthesia & 0 & 0 & \\
Numbness & 0 & 0 & \\
\hline
\end{tabular}

Values are expressed as mean \pm SD or counts. There were no significance differences between groups. PV12: double-injection perivascular ultrasound-guided axillary brachial plexus block at 12 o'clock position of the axillary artery. PV6: double-injection perivascular ultrasound-guided axillary brachial plexus block at 6 o'clock position of the axillary artery. the two groups $(\mathrm{P}=0.630)$. The mean induction time was 543.5 and 579.9 seconds in the PV12 and PV6 group, respectively, so there was no statistical difference $(\mathrm{P}=0.516)$. There was no significant difference between the two groups in the mean number of needle injections (Table 2).

A range of number of axillary arteries and veins were 1-2 and 1-5 respectively in both groups, and there was no difference between the two groups (Table 3 ).

Regarding the sensory block of 4 nerves evaluated for 15 minutes after the block procedure, there was no difference between the two groups in the percentage of patients with a block for all time periods (Fig. 3). Regarding motor block, the comparison of patients with paralysis showed that there was no difference between the two groups for all time periods $(\mathrm{P}>0.05)$ (Fig. 4).

One patient in the PV12 group and 2 patients in the PV6 group had a punctured blood vessel, but the difference was not statistically significant. There were no other symptoms from the toxicity of LA, occurrence of hematoma from blood vessel damage, or complications such as paresthesia or continued numbness caused by nerve damage (Table 3 ).
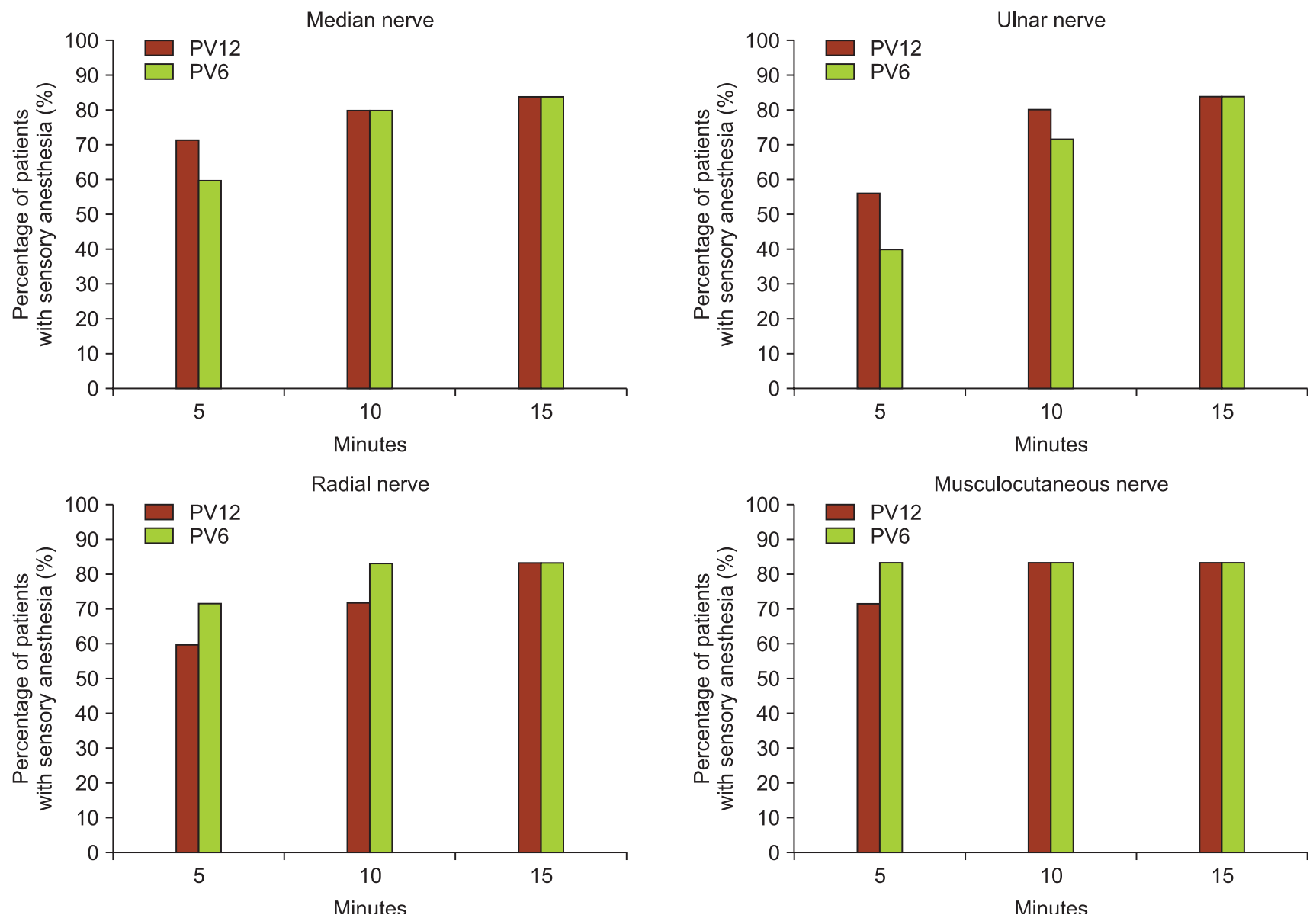

Fig. 3. Percentage of patients with sensory anesthesia (no pinprick) according to time in the cutaneous distributions of the medial, ulnar, radial, and musculocutaneous nerves. There was no statistically significant difference between two groups. 

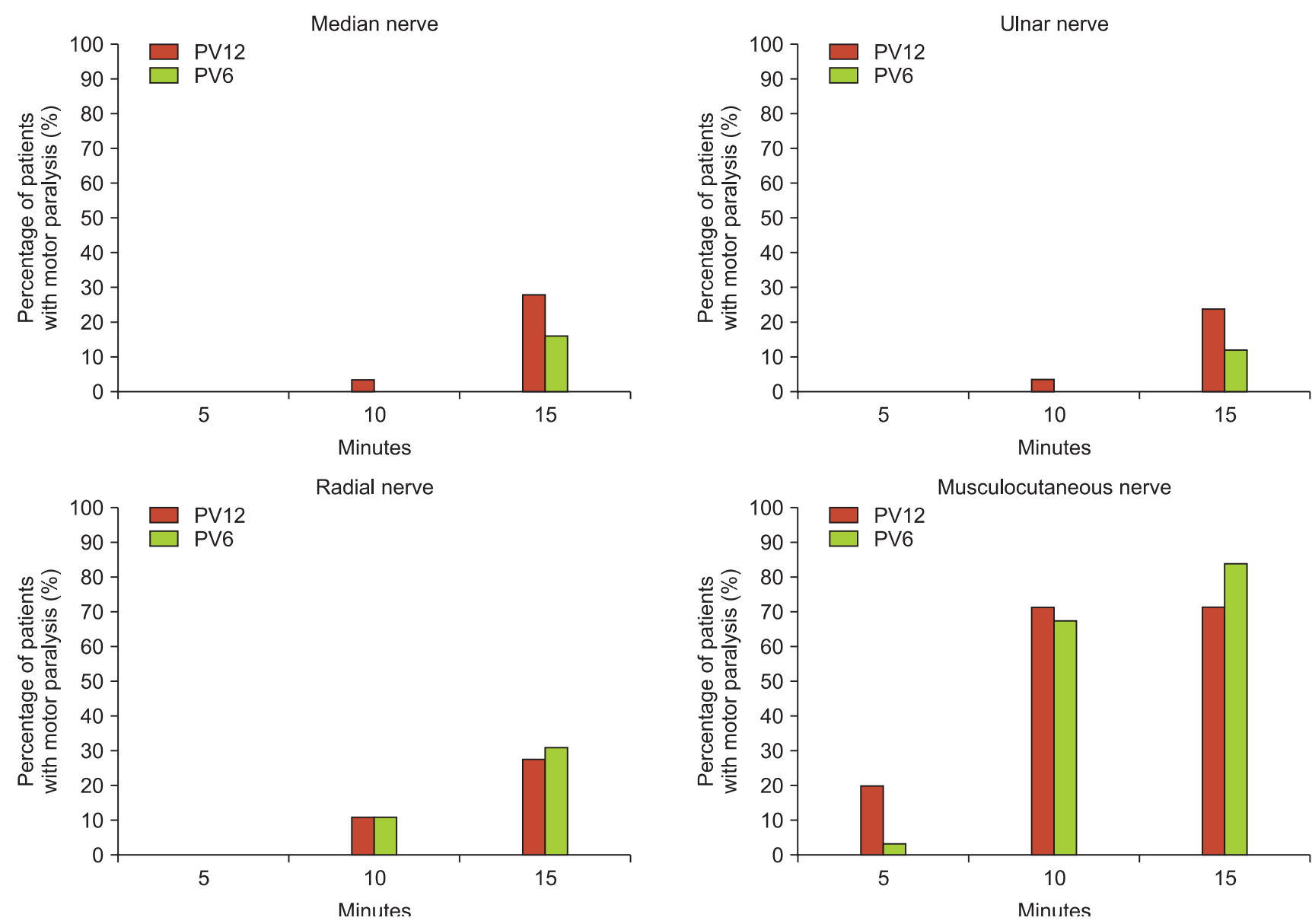

Fig. 4. Percentage of patients with motor paralysis (score of 2) according to time in the distributions of the medial, ulnar, radial, and musculocutaneous nerves. There was no statistically significant difference between two groups.

\section{Discussion}

Our study compared two methods of the perivascular double-injection technique in US-ABPB. The double-injection technique is when the musculocutaneous nerve is separately blocked, and the median nerve, ulnar nerve, and radial nerve are blocked by single injection near the axillary artery. Two groups, which received a single injection in the 6 and 12 o'clock direction of the axillary artery, were compared, and the results showed that there were no differences in the success rate, block performance time, onset time, and induction time.

Many studies have compared PN injection and PV injection in axillary BPB [9-11]. Before the introduction of ultrasound, many studies compared transarterial PV injection and various $\mathrm{PN}$ injection techniques which separately block nerves when performing axillary BPB. From these results, it had been agreed that there was no difference in the success rate between transarterial PV injection and PN injection [12]. Recently, similar studies were conducted using ultrasound $[4,5]$. Imasogie et al. [4] compared a group that was separately blocked median nerve, ulnar nerve, and radial nerve with a group that had LA injected in the 6 o'clock direction of the axillary artery in axillary BPB. The block performance time appeared to be longer in the $\mathrm{PN}$ injection group, and the block score given according to the degree of sensory and motor block appeared to be higher in the PN injection group, but the success rate was not different between the two groups. Bernucci et al. [5] also compared a PN injection group with a group that received PV injection in the 6 o'clock direction of the axillary artery, and the results showed that there were no differences in the success rate and total anesthesia-related time. Tran et al. [13] compared three types of PV injection: only in the 6 o'clock direction, in the 6 and 12 o'clock directions, and in the 2, 10, and 6 o'clock directions. The results showed that there were no differences in the success rate or total anesthesia-related time.

When performing nerve block, it is difficult for LA to spread to areas where the surrounding tissues have high pressure; thus, it is known that in infraclavicular $\mathrm{BPB}$, it is effective to inject the 
LA posterior to the axillary artery where there is high pressure from the pectoral muscle $[14,15]$. In addition to the axillary approach, there have been reports of successful blocks performed by injecting in the 6 o'clock direction [9-11]. However, we thought that because the sheath in the axillary area is surrounded by brachial fascia anteriorly and by latissimus dorsi muscle fascia posteriorly, the pressure will be high enough for both the anterior and posterior directions, so the posterior area of the axillary artery will not be especially superior compared to the other directions. Hence, in PV single injection, the 12 o'clock direction, which was thought to be easier to approach and have less damage to surrounding tissue because it is closer to the skin, was compared with the 6 o'clock direction which was used in precedent studies.

Hickey et al. [16] in the past compared three perivascular techniques without using ultrasound. They reported that when comparing full injection anterior and posterior to the axillary artery and half injection each in the anterior and posterior directions, the posterior injection technique had a significantly lower success rate of median nerve block ( $45 \%$ vs $79-80 \%$; $\mathrm{P}<$ 0.05 ) and a slower onset time (21.5 min vs $11.6-14.5 \mathrm{~min} ; \mathrm{P}<$ $0.05)$. The authors explained that this result was because the median nerve is located anteriorly to the axillary artery, and recommended to avoid full injection in the posterior direction when operating in the area near the distribution of the median nerve.

We identified the relationship of the positions of the axillary artery and nerves, and the topographic anatomy was similar to the research of Christophe et al. [17]. The median nerve was located in the 10-12 o'clock direction in $90 \%$ of the cases, while the ulnar nerve was located in the 1-3 o'clock direction in $96 \%$ of the cases. The radial nerve was located in the 3-5 o'clock direction in $78 \%$ of the cases and in the 6-7 oclock direction in $20 \%$ of the cases. When cases of failure were examined, the radial nerve was not blocked in all 4 examples which failed in the PV12 group, while the median nerve was not blocked in all 4 examples which failed in the PV6 group. Summing up the above, this topographic anatomy can be considered to have an influence on the onset and quality of the block, and nerves closer to the injection position are blocked more quickly and have a higher success rate.

The success rate in our study was $84 \%$ for both groups, which was slightly lower compared to previous studies, such as the research by Bernucci et al. [5] who reported a success rate of 92$96 \%$, Tran et al. [13] reporting a 90-97.5\% success rate, and the research by Imasogie et al. [4] who reported a success rate of 88$89 \%$. Factors that lower the success rate could be not mixing epi- nephrine in the LA, and contrary to precedent studies, the time set for determining success was set as 15 minutes, considering the disinfection and draping process for surgery preparation due to the use of lidocaine which has short duration. In the case of motor block, the percentage of patients with paralysis at 15 minutes was low with $12-32 \%$ in the median nerve, ulnar nerve, and radial nerve, but motor block at the level of paresis was not problematic for performing the surgery.

González et al. [18] recently conducted a study determining the minimum effective volume in double-injection US-ABPB using lidocaine, and they reported that when using $1.5 \%$ lidocaine with epinephrine $5 \mu \mathrm{g} / \mathrm{ml}$, the volume was $23.5 \mathrm{ml}$ for PV and 5.5 $\mathrm{ml}$ for the musculocutaneous nerve. O'Donnell and Iohom [19] reported that when $2 \%$ lidocaine was used in PN injection, anesthesia was achieved with just $1 \mathrm{ml}$ per nerve. The disadvantage of PV injection is that a larger amount of LA could be required compared to PN injection. Bernucci et al. [5] asserted the "silhouette sign" as a method to reduce such waste of LA.

Our research used $24 \mathrm{ml}$ of $2 \%$ lidocaine in PV injection, and observed the separation of the axillary artery and surrounding tissue as a sign of injecting local anesthesia near the blood vessel. In the ultrasound, it was confirmed that the LA was injected in the desired location with the hypoechoic LA accumulating between the artery and surrounding tissue (fascia, nerve, vein, etc). In addition, as the LA diffused, it surrounded the nerves to form a donut sign, and the diffusion of LA to the nerves was confirmed by the nerves appearing to be hyperechoic (Fig. 2).

In both groups, there was no observation of complications such as paresthesia or occurrence of hematoma from vessel puncture. It was predicted that injecting in the 12 o'clock direction would have less damage to the surrounding tissue because it is closer to the skin, but there was no significant difference in vessel puncture, with 1 case in the PV12 and 2 cases in the PV6 group ( $\mathrm{P}=0.552$ ). This is believed to be because the procedure was performed under ultrasound guidance and the surrounding tissue could be avoided.

In conclusion, in the double-injection technique with a single PV injection in US-ABPB, injecting the LA in the 12 o'clock direction and 6 o'clock direction showed no difference in success rate, induction time, and occurrence of adverse events. Hence, the double-injection technique can be performed at 12 o'clock or 6 o'clock position and the needle targeting position can be selected by considering surgery site. Thus, PV double-injection technique is considered to be an alternative method for axillary $\mathrm{BPB}$ even in patients with anatomical variation or difficulties in identifying the individual nerves. 


\section{References}

1. Chan VW, Peng PW, Kaszas Z, Middleton WJ, Muni R, Anastakis DG, et al. A comparative study of general anesthesia, intravenous regional anesthesia, and axillary block for outpatient hand surgery: clinical outcome and cost analysis. Anesth Analg 2001; 93: 1181-4.

2. Casati A, Danelli G, Baciarello M, Corradi M, Leone S, Di Cianni S, et al. A prospective, randomized comparison between ultrasound and nerve stimulation guidance for multiple injection axillary brachial plexus block. Anesthesiology 2007; 106: 992-6.

3. Chan VW, Perlas A, McCartney CJ, Brull R, Xu D, Abbas S. Ultrasound guidance improves success rate of axillary brachial plexus block. Can J Anaesth 2007; 54: 176-82.

4. Imasogie N, Ganapathy S, Singh S, Armstrong K, Armstrong P. A prospective, randomized, double-blind comparison of ultrasound-guided axillary brachial plexus blocks using 2 versus 4 injections. Anesth Analg 2010; 110: 1222-6.

5. Bernucci F, Gonzalez AP, Finlayson RJ, Tran de QH. A prospective, randomized comparison between perivascular and perineural ultrasound-guided axillary brachial plexus block. Reg Anesth Pain Med 2012; 37: 473-7.

6. Thompson GE, Rorie DK. Functional anatomy of the brachial plexus sheaths. Anesthesiology 1983; 59: 117-22.

7. Partridge BL, Katz J, Benirschke K. Functional anatomy of the brachial plexus sheath: implications for anesthesia. Anesthesiology 1987; 66: 743-7.

8. Ay S, Akinci M, Sayin M, Bektas U, Tekdemir I, Elhan A. The axillary sheath and single-injection axillary block. Clin Anat 2007; $20: 57-63$.

9. Koscielniak-Nielsen ZJ, Hesselbjerg L, Fejlberg V. Comparison of transarterial and multiple nerve stimulation techniques for an initial axillary block by $45 \mathrm{ml}$ of mepivacaine $1 \%$ with adrenaline. Acta Anaesthesiol Scand 1998; 42: 570-5.

10. Koscielniak-Nielsen ZJ, Nielsen PR, Nielsen SL, Gardi T, Hermann C. Comparison of transarterial and multiple nerve stimulation techniques for axillary block using a high dose of mepivacaine with adrenaline. Acta Anaesthesiol Scand 1999; 43: 398-404.

11. Turkan H, Baykal B, Ozisik T. Axillary brachial plexus blockade: an evaluation of three techniques. Mil Med 2002; 167: 723-5.

12. De Tran QH, Clemente A, Doan J, Finlayson RJ. Brachial plexus blocks: a review of approaches and techniques. Can J Anaesth 2007; 54: 662-74.

13. Tran de QH, Pham K, Dugani S, Finlayson RJ. A prospective, randomized comparison between double-, triple-, and quadruple-injection ultrasound-guided axillary brachial plexus block. Reg Anesth Pain Med 2012; 37: 248-53.

14. Morimoto M, Popovic J, Kim JT, Kiamzon H, Rosenberg AD. Case series: Septa can influence local anesthetic spread during infraclavicular brachial plexus blocks. Can J Anaesth 2007; 54: 1006-10.

15. Porter JM, McCartney CJ, Chan VW. Needle placement and injection posterior to the axillary artery may predict successful infraclavicular brachial plexus block: a report of three cases. Can J Anaesth 2005; 52: 69-73.

16. Hickey R, Hoffman J, Tingle LJ, Rogers JN, Ramamurthy S. Comparison of the clinical efficacy of three perivascular techniques for axillary brachial plexus block. Reg Anesth 1993; 18: 335-8.

17. Christophe JL, Berthier F, Boillot A, Tatu L, Viennet A, Boichut N, et al. Assessment of topographic brachial plexus nerves variations at the axilla using ultrasonography. Br J Anaesth 2009; 103: 606-12.

18. González AP, Bernucci F, Pham K, Correa JA, Finlayson RJ, Tran de QH. Minimum effective volume of lidocaine for double-injection ultrasound-guided axillary block. Reg Anesth Pain Med 2013; 38: 16-20.

19. O'Donnell BD, Iohom G. An estimation of the minimum effective anesthetic volume of $2 \%$ lidocaine in ultrasound-guided axillary brachial plexus block. Anesthesiology 2009; 111: 25-9. 